\title{
Structural Design and Kinematic Analysis of a Welding Robot for Liquefied Natural Gas Membrane Tank Automatic Welding
}

\author{
Yi Jiang ( $\nabla_{\text {jiangyi@jiangnan.edu.cn ) }}$ \\ Jiangnan University \\ Qingqing Han \\ Jiangnan University \\ Zhaoen Dai \\ Jiangnan University \\ Chao Zhou \\ Jiangnan University \\ Jianfeng Yu \\ Jiangnan University \\ Chunjian Hua \\ Jiangnan University
}

\section{Research Article}

Keywords: Welding robot for membrane tank, Series-parallel hybrid, Screw theory, Kinematic analysis, Working space

Posted Date: January 18th, 2022

DOI: https://doi.org/10.21203/rs.3.rs-1238838/v1

License: (9) This work is licensed under a Creative Commons Attribution 4.0 International License.

Read Full License 


\section{Structural design and kinematic analysis of a welding robot for liquefied natural gas membrane tank automatic welding}

Jiang $\mathrm{Yi}^{1 *} \cdot$ Han Qingqing1 ${ }^{1}$ Dai Zhaoen ${ }^{2} \cdot$ Zhou Chao $^{1} \cdot$ Yu Jianfeng $^{1} \bullet$ Hua Chunjian ${ }^{1}$

1 School of Mechanical Engineering, Jiangsu, Jiangnan University, Wuxi 214000, China

2 School of Vehicle and Mobility, Tsinghua University, Beijing 100084, China

$\square$ Jiang Yi

jiangyi@jiangnan.edu.cn

\section{Acknowledgments}

We thank LetPub (www.letpub.com) for its linguistic assistance during the preparation of this manuscript. 


\begin{abstract}
Owing to the complex structures of welding materials, special welding conditions, and challenges during the automatic welding of the liquefied natural gas (LNG) ship Mark III's membrane tank, a series-parallel-series hybrid structure mobile welding robot having sufficient adaptability for welding corrugated plates in membrane tanks was designed in this study. The configuration of the hybrid robot had good workspace characteristics, and it could always maintain a certain distance and angle between the end of the welding torch and the weld line because of its detection and control system coordination. In this study, degrees of freedom, kinematic characteristics, workspace, and adaptability analyses were conducted for the hybrid mechanism. A simulation verification was performed, and a ripple-trajectory-following experiment was conducted using real objects. The simulation and experimental results showed that the welding robot had a reasonable mechanism design, smooth motion, and good terminal distance and angle control, thus meeting the requirements for automatic welding of corrugated plates in membrane tanks.

Keywords Welding robot for membrane tank $\bullet$ Series-parallel hybrid $\bullet$ Screw theory $\bullet$ Kinematic analysis • Working space
\end{abstract}




\section{Introduction}

Liquid cargo enclosure technology is the core technology in liquefied natural gas (LNG) ships, and membrane cargo tanks have increasingly been used because of their advantageous characteristics, including their strong shock resistance ability, high space utilization rate, low evaporation, and low maintenance costs.

A membrane cargo tank is a large, fully enclosed structure with a height of up to 15 meters. The bulkhead is a sandwich-type multi-layer structure. The main barrier wall film consists of welded 304L corrugated austenitic stainless-steel plates with thicknesses of $1.2 \mathrm{~mm}$. There are latticed grooves of different sizes on the film, which are folded at the intersections and have special shapes [1]. In addition, membrane cargo tanks have high product welding quality requirements for the sake of security [2]. As the main way of corrugated plate welding for membrane tank at this stage, the manual tungsten inert gas welding (TIG) does not perform well. It is also necessary to build an aerial platform during the manual TIG process for LNG membrane tanks. This is not only complicated but also inefficient; it introduces a high-risk factor, and ensuring quality becomes a challenge.

Conventional robotic welding systems are seldom used because shipyard welding tasks are characterized by non-standard, large workpieces that come in small batches [3]. At present, studies investigating automatic robotic welding primarily focus on weld-track recognition and tracking. For example, Zhou et al. [4] proposed a welding seam sensing method based on a line-structured light sensor and constructed an intelligent guidance system for a welding robot. Li et al. [5] designed a dual-station intelligent welding strategy based on charge-coupled device (CCD) visual detection system. Zhang et al. [6] proposed a four-step welding seam tracking technology for complex welds based on laser sensors. Special automatic welding equipment $[7,8]$, particularly 
the special equipment related to shipbuilding welding $[9,10]$, has rarely been studied. In addition, the welds in membrane tanks were always long and high above the ground, which was beyond the working space of the existing automatic welding equipment. Therefore, there is great significance and practical value in developing automatic welding equipment suitable for membrane chambers instead manual welding.

Three problems should be solved for automatic welding of corrugated plates in membrane tanks:

1. The end of the TIG welding torch should maintain a certain distance and angle with respect to the weld.

2. The shape change of a corrugated plate should be accurately detected, and each motion pair must quickly follow the linkage with good stiffness and precision.

3. Sufficient working space characteristics are required, and the length of a continuous weld should be greater than $1.5 \mathrm{~m}$.

For these three problems, a series-parallel-series hybrid mobile welding robot structure scheme with good adaptability is proposed in this paper for membrane tank welding. A kinematics analysis was conducted, the adaptability of the workspace to the working conditions was investigated, and simulations were performed. Additionally, a corrugated trajectory-following experiment was conducted that demonstrated the rationality and feasibility of the design.

\section{Robot structure}

The proposed structure of an automatic welding robot for membrane tanks [11] and its three-dimensional model are shown in Fig. 1. To adapt to work high above the ground and to promptly change the working position, an overall lightweight design was desirable. The 6061 aluminum alloy was selected as the robot body material, the body base was $270 \times 280 \mathrm{~mm}$, the 
total height was $350 \mathrm{~mm}$, and the body mass was only $13 \mathrm{~kg}$. These characteristics met the requirements for rapid assembly and disassembly and a lightweight design.

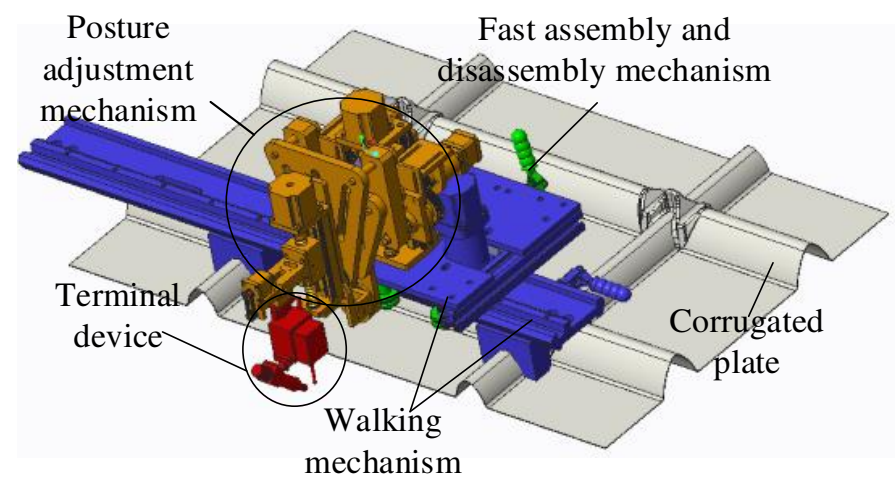

Fig. 1 Working diagram of the robot in a flat welding condition

The robot consisted of four detachable modules: a posture-adjustment mechanism, a terminal device, a walking mechanism, and a rapid assembly and disassembly mechanism. The first two modules constituted the robot body. The posture-adjustment mechanism could change the position and orientation of the robot terminal device. The walking mechanism was composed of a guide rail and a transmission mechanism, which could constrain the robot to the welding working surface and could move along the direction of the weld. The rapid assembly and disassembly mechanism enabled swift separation and combination of the body and the walking mechanism.

The overall design of the robot orientation-adjustment mechanism's motion chain had a series-parallel-series transmission form, as shown in Fig. 2. The fixed platform of the parallel mechanism (6) was mounted on the $Y$-axis slide block (7) and moved in the $Y$-direction through the guide screw. $V$-axis (2) and $X$-axis (1) screws were mounted in series on the parallel mechanism's moving platform (9). The entire motion chain mechanism adjusted the position and orientation of the terminal device by the coordination of the $Y$-axis, fixed and moving platform of parallel mechanism, and the $V$ - and $X$-axis screws (series-parallel-series). 


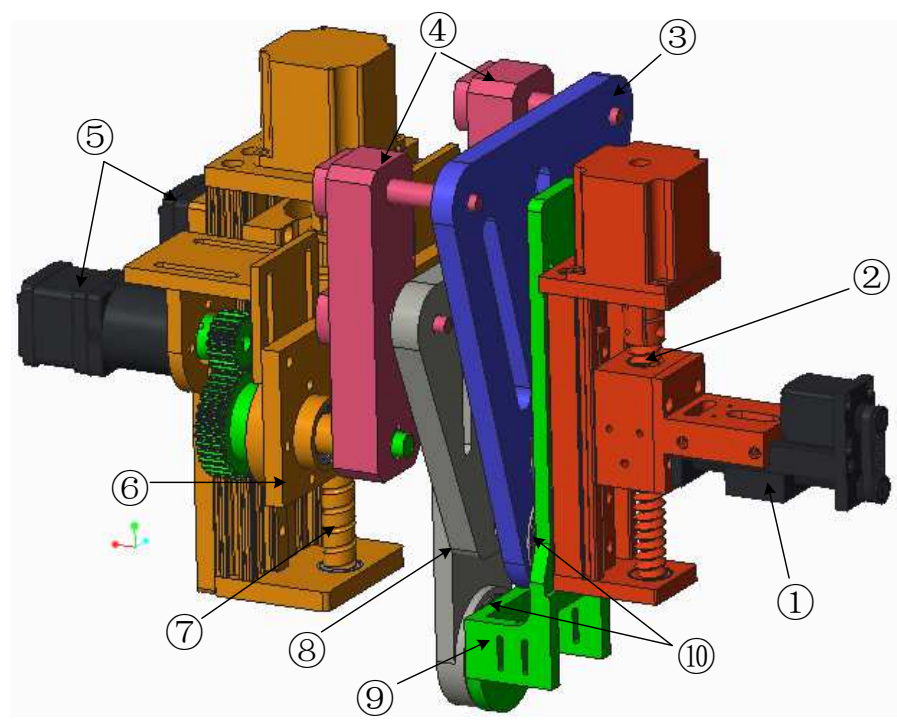

Fig. 2 Position and orientation-adjusting mechanism: $1 \mathrm{X}$-axis guide screw, $2 \mathrm{~V}$-axis guide screw, 3 front connection plate, 4 Big arm, 5 A-axis driving motor,6 parallel fixed platform,7 Y-axis guide screw, 8 back connection plate, 9 parallel linkage platform, 10 crossed roller bearing

The series-parallel-series hybrid design improved the stiffness and bearing capacity of the mechanism while ensuring its mobility and dexterity. In addition, a linear variable differential transformer (LVDT) sensor set placed at the end caused the welding torch end to maintain a certain distance from the workpiece and caused the welding torch axis and welding spot section to maintain a certain angle to meet the TIG welding process requirements. Problems 1 and 2 from Section 1 must be solved at the mechanism design level.

To solve Problem 3, that is, that the robot had a small arm span, a limited working space, and could not perform long welds, and to account for the man-machine combination efficiency, the walking mechanism and the rapid assembly and disassembly mechanism were designed as shown in Fig. 3. A closed-loop stepping motor rack, a pinion drive mechanism (9), (6), (8), a roller (3), and a guide rail (7) cooperated with each other to position and guide the robot body. A self-locking rapid fixture (5) pushed the sliding roller baseplate (4), and drove the trapezoidal 
groove roller to buckle the guide rail surface to achieve swift locking. After completing a long weld, the robot body must only be placed on the guide rail, which would be pre-clamped at the fold of the membrane corrugated plate, and the self-locking rapid fixture could quickly switch between flat welding, vertical welding, horizontal welding, and inverted welding.

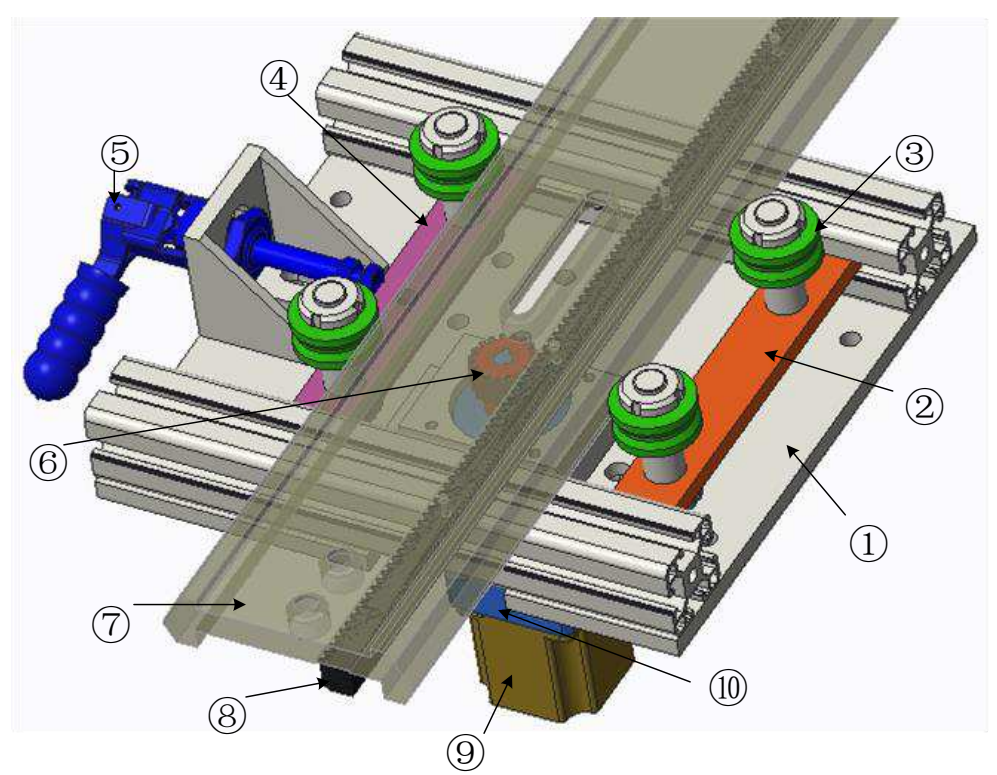

Fig. 3 Walking mechanism and rapid assembly and disassembly mechanism diagram:1 body baseplate, 2 fixed side roller baseplate, 3 trapezoidal groove roller, 4 sliding side roller baseplate, 5 rapid fixture, 6 gear, 7 guide rail, 8 rack, 9 closed-loop stepper motor, 10 planet-gear speed reducer

\section{Kinematic analysis}

\subsection{Degree-of-freedom analysis}

According to a further analysis of the requirements for Problem 1, to ensure sufficient welding quality during membrane tank corrugated plate welding, the welding torch must be adjustable in the length, width, and depth directions, and must be capable of adapting to the dynamic angle 
between the welding torch and the welding surface curvature. Therefore, at least one rotational and three mobile degrees of freedom were required, and a mixed degree-of-freedom (DOF) analysis must be performed.

The robot designed had a hybrid configuration, and the DOF total was equal to the DOF sum for the series and parallel portions. There were four degrees of freedom for the four translational axes in the series portion. The parallel structure was relatively complex; to determine its degrees of freedom, an equivalent analysis was first done by observing its structural characteristics, as shown in Fig. 4a.

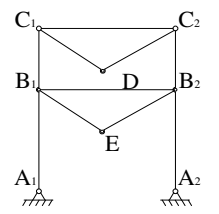

(a)

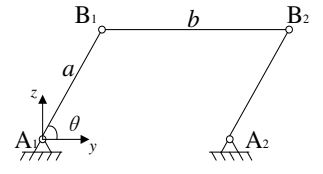

(b)

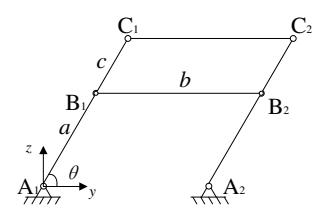

(c)

Fig. 4. Equivalent mechanism diagram. a Equivalent diagram; b parallelogram mechanism; c Expander graph

The DOF analysis after equivalence was performed based on screw theory, and the modified Kutzbach-Gubler formula [12] was adopted for the analysis:

$$
M=6(n-g-1)+\sum_{i=1}^{g} f_{i}+\mu
$$

Due to the common motion pairs on the two branch chains of the mechanism, the coupling strength was relatively high, and consequently, a direct analysis was difficult. For this reason, the simple four-bar mechanism shown in Fig. $4 \mathrm{~b}$ was analyzed first. The mechanism was regarded as a parallel mechanism composed of the $\mathrm{A}_{i} \mathrm{~B}_{i}(i=1,2)$ branch and the output rod, $\mathrm{B}_{1} \mathrm{~B}_{2}$. The $\mathrm{A}_{1} \mathrm{~B}_{1}$ branch chain motion screw system could be represented by Eq. 2: 


$$
\left\{\begin{array}{l}
\boldsymbol{S}_{A I}=(1,0,0 ; 0,0,0) \\
\boldsymbol{S}_{B I}=(1,0,0 ; 0,-a \sin \theta, a \cos \theta)
\end{array} .\right.
$$

Its constraint screw system is given by Eq. 3:

$$
\left\{\begin{array}{l}
\boldsymbol{S}_{11}^{\mathrm{r}}=(1,0,0 ; 0,0,0) \\
\boldsymbol{S}_{12}^{\mathrm{r}}=(0,0,0 ; 0,1,0) \\
\boldsymbol{S}_{13}^{\mathrm{r}}=(0,0,0 ; 0,0,1) \\
\boldsymbol{S}_{14}^{\mathrm{r}}=(0, \cos \theta, \sin \theta ; 0,0,0)
\end{array} .\right.
$$

The $\mathrm{A}_{2} \mathrm{~B}_{2}$ branch chain motion screw system can be expressed by Eq. 4:

$$
\left\{\begin{array}{l}
\boldsymbol{S}_{A 2}=(1,0,0 ; 0,0, b) \\
\boldsymbol{S}_{\boldsymbol{B} 2}=(1,0,0 ; 0,-a \sin \theta, b+a \cos \theta)
\end{array} .\right.
$$

Its constraint screw system is given by Eq. 5:

$$
\left\{\begin{array}{l}
\boldsymbol{S}_{21}^{\mathrm{r}}=(1,0,0 ; 0,0,0) \\
\boldsymbol{S}_{22}^{\mathrm{r}}=(0,0,0 ; 0,1,0) \\
\boldsymbol{S}_{23}^{\mathrm{r}}=(0,0,0 ; 0,0,1) \\
\boldsymbol{S}_{24}^{\mathrm{r}}=(0, \cos \theta, \sin \theta ;-b \sin \theta, 0,0)
\end{array} .\right.
$$

The mechanism in Fig. $4 \mathrm{~b}$ had eight constraints, among which three were over-constraints; therefore, the screw system of the mechanism constraint is given by Eq. 6 :

$$
\left\{\begin{array}{l}
\boldsymbol{S}_{1}^{\mathrm{c}}=(1,0,0 ; 0,0,0) \\
\boldsymbol{S}_{2}^{\mathrm{c}}=(0,0,0 ; 0,1,0) \\
\boldsymbol{S}_{3}^{\mathrm{c}}=(0,0,0 ; 0,0,1) \\
\boldsymbol{S}_{4}^{\mathrm{c}}=(0, \cos \theta, \sin \theta ;-b \sin \theta, 0,0)
\end{array} .\right.
$$

The output rod, $\mathrm{B}_{1} \mathrm{~B}_{2}$, of the mechanism had a single degree of freedom, and the motion direction was always perpendicular to $A_{1} B_{1}$. For further analysis, the mechanism shown in Fig. $4 c$ added a parallel rod, $C_{1} C_{2}$, above the mechanism shown in Fig. $4 \mathrm{~b}$. It is worth noting that $C_{1}$ and $C_{2}$ were located on $A_{1} B_{1}$ and $A_{2} B_{2}$, respectively, and therefore, the motion pattern $C_{1}$ and $C_{2}$ was 
consistent with $A_{1} B_{1}$ and $A_{2} B_{2}$, respectively. Considering the influence of $C_{1} C_{2}$ on the overall constraint, the motion spiral system at $\mathrm{C}_{1} \mathrm{C}_{2}$ can be expressed by Eq. 7 :

$$
\left\{\begin{array}{l}
\boldsymbol{S}_{C 1}=(1,0,0 ; 0,-(a+c) \sin \theta,(a+c) \cos \theta) \\
\boldsymbol{S}_{C 2}=(1,0,0 ; 0,-(a+c) \sin \theta, b+(a+c) \cos \theta) .
\end{array}\right.
$$

Taking the inverse helix resulted in Eq. 8:

$$
\left\{\begin{array}{l}
\boldsymbol{S}_{31}^{\mathrm{r}}=(1,0,0 ; 0,0,0) \\
\boldsymbol{S}_{32}^{\mathrm{r}}=(0,0,0 ; 0,1,0) \\
\boldsymbol{S}_{33}^{\mathrm{r}}=(0,0,0 ; 0,0,1) \\
\boldsymbol{S}_{34}^{\mathrm{r}}=(0, \cos \theta, \sin \theta ;-b \sin \theta, 0,0) .
\end{array}\right.
$$

Thus, the addition of $\mathrm{C}_{1} \mathrm{C}_{2}$ brought no additional independent constraints, but only four over-constraints. Similarly, as the mechanism in Fig. 4a was equivalent to adding a rod, DE, parallel to $\mathrm{B}_{2} \mathrm{C}_{2}$ on the mechanism in Fig. $4 \mathrm{c}$, and because $\Delta \mathrm{C}_{1} \mathrm{C}_{2} \mathrm{D} \cong \Delta \mathrm{B}_{1} \mathrm{~B}_{2} \mathrm{E}$, the rod brought four over-constraints. Therefore, the mechanism had a total of 11 over-constraints, and according to Eq. 1:

$$
M=6(n-g-1)+\sum_{i=1}^{g} f_{i}+\mu=6 \times(6-8-1)+8+11=1 .
$$

Therefore, the parallel mechanism had one degree of freedom, which was the motion of $\mathrm{B}_{1} \mathrm{~B}_{2}$ perpendicular to $A_{1} B_{1}$, i.e., the rotations of $D E$ and $A_{1} C_{1}$ were consistent. Including the series portion, the hybrid mechanism had a total of five degrees of freedom with four translational shifts and one rotation, which met the DOF welding requirements.

\subsection{Motion law analysis of the parallel mechanism}

The DOF analysis of the mechanism from Section 3.1 indicated that the parallel mechanism achieved the rotational degree of freedom of the moving platform, DE. However, in general, if there is an uncertain rotational center when a mechanism rotates, points on the moving platform 
will move in non-DOF directions during rotation $[13,14]$. This motion in the non-DOF directions affects the motion flexibility during welding and thus is not conducive to weld control and formation. Therefore, it was necessary to analyze the motion law of the parallel mechanism. Since the parallel mechanism was compact in structure and had few degrees of freedom, the spatial geometry method was used for analysis.

To study a more general rule, a more general situation was considered. The parallel mechanism could be represented as an equivalent structure, as shown in Fig. 5a. $\Delta \mathrm{C}_{1} \mathrm{C}_{2} \mathrm{D}$ and $\Delta \mathrm{B}_{1} \mathrm{~B}_{2} \mathrm{E}$ were regarded as rigid connections, $\mathrm{A}_{1} \mathrm{C}_{1}$ and $\mathrm{A}_{2} \mathrm{C}_{2}$ as rigid bars, and $\mathrm{C}_{2} \mathrm{D} \neq \mathrm{B}_{2} \mathrm{E}$. The coordinate system $A_{1} x y z$ was established at $\mathrm{A}_{1}$ and the parallel part of the mechanism was projected onto the $x A_{1} z$ plane, as shown in Fig. $5 b$.

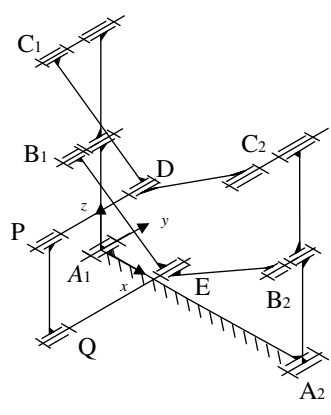

(a)

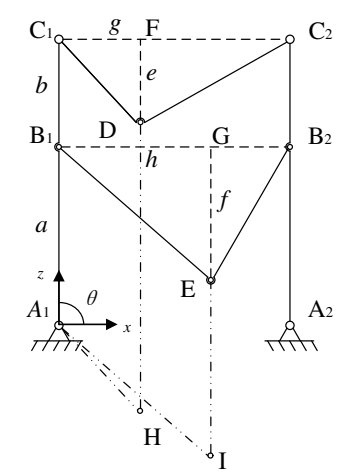

(b)

Fig. 5 Diagram of the parallel mechanism. a Brief diagram of the parallel mechanism; b Projection onto the $x A_{1} z$ plane

In Fig. 5, $\mathrm{A}_{1} \mathrm{~B}_{1}=\mathrm{A}_{2} \mathrm{~B}_{2}=a, \mathrm{~B}_{1} \mathrm{C}_{1}=\mathrm{B}_{2} \mathrm{C}_{2}=b, \mathrm{DF}=e, \mathrm{EG}=f, \mathrm{C}_{1} \mathrm{~F}=g, \mathrm{~B}_{1} \mathrm{G}=h$,

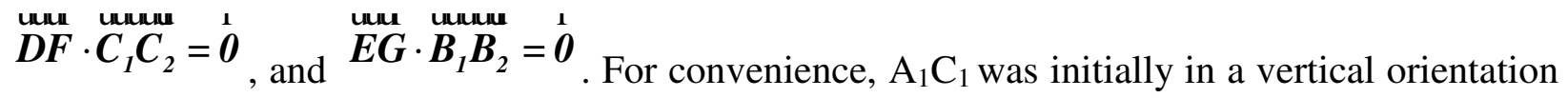
and the driving force was around the $y$-axis of coordinate system $\left\{A_{1}\right\}$, so that $\mathrm{A}_{1} \mathrm{C}_{1}$ and $x$ had the angle $\theta$ between them. As the $\mathrm{C}_{1}$ coordinate was set as $\left(x_{\mathrm{C} 1}, z_{\mathrm{C} 1}\right)$, the trajectory of $\mathrm{C}_{1}$ could be expressed by Eq. 9: 


$$
x_{\mathrm{C} 1}^{2}+\mathrm{z}_{\mathrm{C} 1}^{2}=(a+b)^{2} \text {. }
$$

The quadrilateral $\mathrm{A}_{1} \mathrm{~A}_{2} \mathrm{C}_{2} \mathrm{C}_{1}$ formed a parallelogram, hence at any time ${\stackrel{C}{1} \boldsymbol{C}_{2}}_{2}=\boldsymbol{A}_{1} \boldsymbol{A}_{2}$. Eq. 10 was true for coordinate system $\left\{A_{1}\right\}$ :

$$
\left(x_{\mathrm{D}}, z_{\mathrm{D}}\right)^{\mathrm{T}}=\left(x_{\mathrm{C} 1}+g, z_{\mathrm{C} 1}-e\right)^{\mathrm{T}} .
$$

Then, Eq. 11 could be obtained from Eqs. 9 and 10:

$$
\left(x_{\mathrm{D}}-g\right)^{2}+\left(z_{\mathrm{D}}+e\right)^{2}=(a+b)^{2} \text {. }
$$

According to Eq. 11, while the mechanism was moving, the rotation pair D moved in a circle around a fixed axis in space. If the projection of the axis in the $A_{1} x z$ plane was $\mathrm{H}(g,-e)$, Eq. 5 could be generated using Eq. 11:

$$
\mathrm{DH}=a+b=\mathrm{A}_{1} \mathrm{C}_{1} .
$$

The distance from $\mathrm{H}$ to coordinate system $\left\{A_{1}\right\}$ could be expressed by Eq. 13:

$$
\mathrm{A}_{1} \mathrm{H}^{2}=g^{2}+e^{2}
$$

For the right triangle $\mathrm{C}_{1} \mathrm{DF}$, Eq. 14 was true:

$$
\mathrm{C}_{1} \mathrm{D}^{2}=g^{2}+e^{2}
$$

According to Eqs. $12-14$, the quadrilateral $\mathrm{A}_{1} \mathrm{C}_{1} \mathrm{DH}$ was a parallelogram, and therefore $\mathrm{HD} \| \mathrm{A}_{1} \mathrm{C}_{1}$, that is, the rotational pair $\mathrm{D}$ had the same angular velocity as the rigid $\operatorname{rod} \mathrm{A}_{1} \mathrm{C}_{1}$ when rotating around the axis. Therefore, the coordinates of $\mathrm{D}$ were $(g+(a+b) \cdot \cos \theta, e+(a+b) \cdot \sin \theta)$. Similarly, the rotational pair E rotated about a fixed axis in space and had the same angular velocity as rod $\mathrm{A}_{1} \mathrm{C}_{1}$. The projection of the axis in the $A_{1} x y$ plane was $\mathrm{I}(h,-f)$, and the trajectory equation for the rotational pair $\mathrm{E}$ about the axis was $\left(x_{\mathrm{E}}-h\right)^{2}+\left(\mathrm{z}_{\mathrm{E}}+f\right)^{2}=a^{2}$. Similarly, for the rotational pair $\mathrm{E}$, the coordinates of $\mathrm{E}$ were $(h+a \cdot \cos \theta, f+a \cdot \sin \theta)$. When $\mathrm{A}_{1} \mathrm{C}_{1}$ was at any angle $\theta$ with respect to $x$, the moving platform ED was at an angle $\alpha$ with respect to $x$, and Eqs. 15 and 16 were true: 


$$
\begin{gathered}
\mathrm{ED}=\left((g-h+b \cos \theta)^{2}+(e-f+b \sin \theta)^{2}\right)^{1 / 2}, \\
\alpha=\arctan \frac{(e-f)+b \sin \theta}{(g-h)+b \cos \theta} .
\end{gathered}
$$

According to Eqs. 15 and 16, the moving platform rotated with the rotation of $A_{1} C_{1}$, and the distance between joints D and E changed accordingly.

\subsection{Forward and inverse kinematics analyses}

This section presents further analyses of the requirements for Problem (2). Although an LVDT sensor group was used to detect the instantaneous position and orientation of the welding gun, it was still necessary to conduct kinematics forward and inverse solution analyses to cause each motion pair to quickly follow the linkage and achieve the desired position and orientation. Kinematics analyses are not only the basis of robot motion control, but also the basis of subsequent workspace analysis [15].

According to the analysis in Section 3.2, the rotation angle and length of ED on the moving platform changed nonlinearly with the rotation of $\mathrm{A}_{1} \mathrm{C}_{1}$. Such a change increased the complexity of the mechanism and control algorithm. Eqs. 15 and 16 indicate that if $e=f$ and $g=h$, then $\alpha=\theta$ and the length of ED was a constant value. At this point, the parallel mechanism was considered equivalent to a virtual revolute joint existing outside the mechanism. The motion of the parallel mechanism's moving platform was regarded as circular motion around the revolute joint, and the motion law was consistent with that of the big arm $\mathrm{A}_{1} \mathrm{C}_{1}$, which caused the system complexity to decrease. The results and analyses in the rest of the paper were based on $e=f$ and $g=h$.

First, the direct kinematics were studied, that is, the mapping between the robot joint space and the Cartesian coordinate space. To facilitate the analysis, each joint in the mechanism was 
adjusted to a special horizontal or vertical position, and the coordinate system was established according to the D-H model criterion [16]. The equivalent hybrid mechanism and coordinate system are shown in Fig. 6, and the values of each parameter in the D-H model are listed in Table 1.

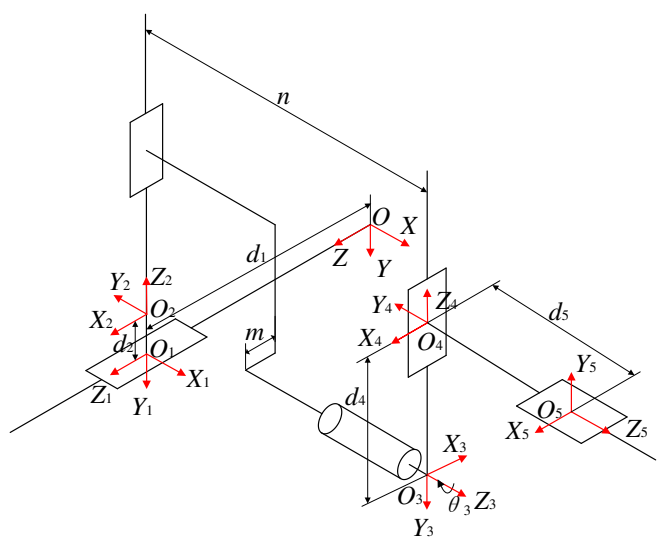

Fig. 6 Equivalent simplified model of the five-DOF hybrid mechanism

Table $1 \mathrm{D}-\mathrm{H}$ matrix parameter list

\begin{tabular}{lllll}
\hline $\begin{array}{l}\text { Joint number } \\
i\end{array}$ & $\begin{array}{l}\text { Link length } a_{\mathrm{i}-1} \\
(\mathrm{~mm})\end{array}$ & $\begin{array}{l}\text { Twist angle } \alpha_{\mathrm{i}-1} \\
\left({ }^{\circ}\right)\end{array}$ & $\begin{array}{l}\text { Link offset } d_{i} \\
(\mathrm{~mm})\end{array}$ & $\begin{array}{l}\text { Link angle } \theta_{i} \\
\left({ }^{\circ}\right)\end{array}$ \\
\hline 1 & 0 & 0 & $d_{1}$ & 0 \\
2 & 0 & 90 & $d_{2}$ & 90 \\
3 & $m$ & 90 & $n$ & $180+\theta_{3}$ \\
4 & 0 & 90 & $d_{4}$ & 180 \\
5 & 0 & 90 & $d_{5}$ & 0 \\
\hline \multicolumn{5}{r}{ In the D-H model modified based on Craig's rule, the general transformation matrix } \\
expression for the coordinate system $\{i-1\}$ relative to the coordinate system $\{i\}$ is given in Eq. 17:
\end{tabular}




$$
{ }^{i-1} T_{i}=\left[\begin{array}{cccc}
\cos \theta_{i} & -\sin \theta_{i} & 0 & a_{i-1} \\
\sin \theta_{i} \cos \alpha_{i-1} & \cos \theta_{i} \cos \alpha_{i-1} & -\sin \alpha_{i-1} & -d_{i} \sin \alpha_{i-1} \\
\sin \theta_{i} \sin \alpha_{i-1} & \cos \theta_{i} \sin \alpha_{i-1} & \cos \alpha_{i-1} & d_{i} \cos \alpha_{i-1} \\
0 & 0 & 0 & 1
\end{array}\right] .
$$

The matrix transformation of the robot end actuator relative to the base system could be expressed by Eq. 18:

$$
{ }^{0} T_{n}=\prod_{i=1}^{n}{ }^{i-1} T_{i} .
$$

The forward kinematics problem was to find the position and orientation of the manipulator relative to the base robot system, namely, to find the homogeneous transformation matrix ${ }^{0} T_{5}$. By substituting the D-H parameters from Table 1 into Eq. 17, the homogeneous transformation matrix ${ }^{i-1} T_{i}$ for each joint was obtained. According to Eq. 18, the forward kinematics solution could be expressed by Eq. 19:

$$
{ }^{0} T_{5}=\left[\begin{array}{cccc}
n_{x} & o_{x} & a_{x} & p_{x} \\
n_{y} & o_{y} & a_{y} & p_{y} \\
n_{z} & o_{z} & a_{z} & p_{z} \\
0 & 0 & 0 & 1
\end{array}\right] .
$$

In Eq. 19, $a_{x}=1, n_{y}=-s_{3}, o_{y}=-c_{3}, n_{z}=c_{3}, o_{z}=s_{3}, p_{x}=d_{5}+n, p_{y}=-d_{2}-d_{4} c_{3}, p_{z}=d_{4} s_{3}+d_{1}+m$, $n_{\mathbf{x}}=o_{x}=a_{y}=a_{z}=0$.

Then the inverse kinematics solution was studied, that is, the mapping relationship between the Cartesian coordinate system and the joint coordinate system. According to the above analysis, the walking mechanism expanded the welding working space of the robot and introduced a redundant degree of freedom. Therefore, countless sets of solutions must exist for each end actuator orientation. Since the orientation-adjustment mechanism of the robot body had the required four-DOF orientation-adjustment capability, the displacement of the walking mechanism 
could be regarded as the translation expansion of the robot's motion space in practical applications. Therefore, the robot motion was decoupled from the motion of the four-DOF robot along the walking axis. This study analyzed the inverse solution for the robot when walking to any position, that is, it assumed that any $d_{1}$ was a constant and $d_{1} \in\left(d_{1 \min }, d_{1 \max }\right)$. According to the kinematics analysis of the parallel mechanism, $\theta_{3}$ was the actual orientation angle of the end actuator, that is, $\alpha$ $=\theta_{3}$. The inverse kinematics equation was then obtained according to Eq. 19:

$$
\left\{\begin{array}{l}
d_{2}=-p_{y}-\left(p_{x}-m-d_{1}\right) \cot \theta_{3} \\
d_{4}=\frac{p_{z}-m-d_{1}}{\sin \theta_{3}} \\
d_{5}=p_{x}-n \\
\theta_{3}=\alpha
\end{array}\right.
$$

\subsection{Workspace analysis}

This section presents further analysis of the requirements of Problem 3. Although the walking mechanism met the working space requirements in the weld length direction, one of the difficulties in welding membrane corrugated stainless-steel is that stamping and corrugated size errors are always present. Additionally, the film surfaces are highly reflective, which causes difficulty in conducting real-time welding trajectory detection and compensation using optical means. In this case, to achieve high welding accuracy, it is very important to adequately match the workspace characteristics of the welding robot and the thin-film corrugated surface structure.

The robot workspace is an important kinematic index reflecting the robot's working characteristics and the applicability of the working conditions; it also verifies the rationality of the mechanism design to a certain extent [17]. The ripple position is the key welding position. The Monte Carlo method [18] was used to investigate the motion space of the welding robot with the ripple position as the reference. The implementation process consisted of four primary steps: 
(a) The range of values for each joint variable were determined $\left(\theta_{i \min }, \theta_{i \max }\right)$.

(b) $N$ groups of random value sequences of joint variables were generated by random functions $\left\{\theta_{i} \mid \theta_{i}=\theta_{i \min }+\left(\theta_{i \max }-\theta_{i \min }\right) * R A N D\right\}$, where $i=1,2,3,4,5$ and $R A N D$ is a random number between 0 and 1 .

(c) By substituting the joint variables, $\theta_{i}(i=1,2,3,4,5)$, into the orientation transformation matrix, ${ }^{0} T_{5}$, the position vectors of the mechanism's end actuator, $\boldsymbol{P}=\left[P_{X}, P_{Y}, P_{Z}\right]$, were obtained.

(d) A three-dimensional scatter diagram was drawn using MATLAB, and the spatial point cloud of the robot's terminal motion was obtained.

The walking axis, $Z_{1}$, was set to be stationary, and the workspace distribution of the robot body was studied. Within the value range of each joint variable, the robot workspace obtained using the Monte Carlo method is shown in Fig. 7.

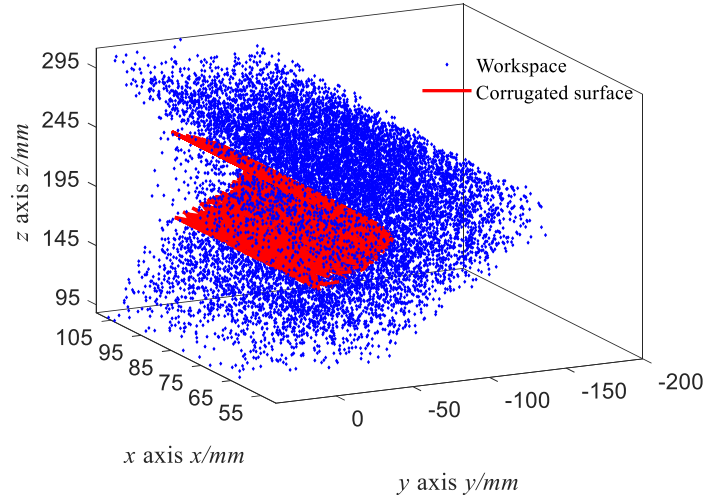

(a)

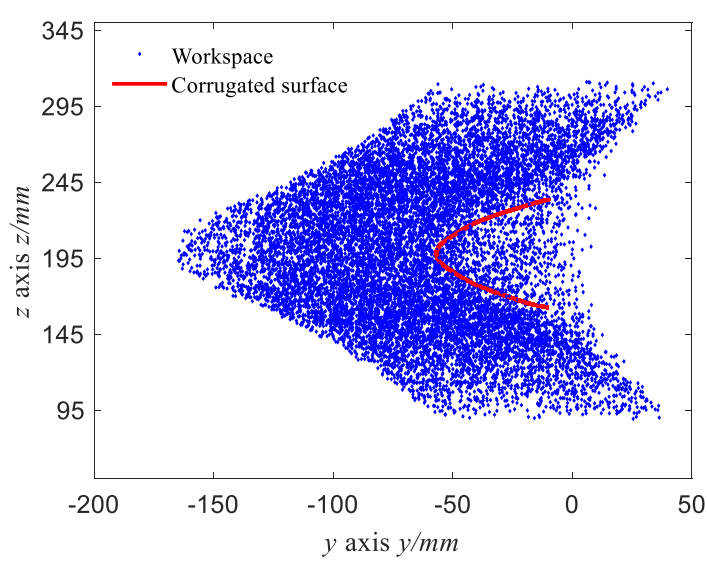

(b)

Fig. 7 Cloud of points for the manipulator workspace. a Three-dimensional view of the robot workspace; b $Y O Z$ view

In this figure, the $x, y$, and $z$ directions correspond to the width, depth, and length directions, respectively, of the weld during the welding process. Fig. 7a shows that the contour of the robot's working space was of a curved-roof type, and the motion range of the welding gun in the width 
direction was $70 \mathrm{~mm}$, which meets the welding requirements of the folded structure $(40 \mathrm{~mm})$. Fig. $7 \mathrm{~b}$ shows that the workspace section shape corresponded to the shape of the corrugated plate. When the wave peak of the corrugated plate and the virtual robot rotational center were at the same position along the $z$-axis, the allowable welding radius of the corrugated plate was larger than the standard corrugated size and had a large workspace redundancy. The workspace size and corrugated-curve structure were well matched.

\section{Kinematic simulations}

\subsection{Degree-of-freedom verification}

The kinematic pair and drive were added to the robot model, and a mechanism model verification using Automatic Dynamic Analysis of Mechanical System (ADAMS) software showed that the parallel mechanism had one degree of freedom, while there were five degrees of freedom in total for the hybrid mechanism. The mechanism had 11 over-constraint equations, which were consistent with the screw theory analysis results.

\subsection{Forward kinematics verification}

To verify the correctness of the forward kinematics solution, the offset distance between the virtual rotational center and the symmetric center of the mechanism was set to $m=0$, and $n=171 \mathrm{~mm}$ was also selected. Forward and backswing motions with 20 -second periods and 10 -second lengths were implemented, and the trajectory of each moving joint could be described by Eq. 21: 


$$
\left\{\begin{array}{l}
d_{1}=100 \sin \left(\frac{\pi}{10} \cdot t\right) \\
d_{2}=50 \sin \left(\frac{\pi}{10} \cdot t\right) \\
\theta_{3}=60 \sin \left(\frac{\pi}{10} \cdot t\right) \\
d_{4}=20 \sin \left(\frac{\pi}{10} \cdot t\right) \\
d_{5}=20 \sin \left(\frac{\pi}{10} \cdot t\right)
\end{array} .\right.
$$

By substituting Eq. 21 into the kinematics forward solution model in Eq. 19, the theoretical calculated position data for the end mechanism in the $X, Y$, and $Z$-directions relative to the base coordinate system were obtained. Additionally, the drive shown in Eq. 21 was also applied to each joint in the ADAMS model to simulate the robot's motion state. After unifying the post-simulation processing data and the theoretical calculation data, the results shown in Fig. 8 were obtained. These figures show that the simulation data and the data obtained by theoretical calculation had the same variation trends and that the error was very small, thus verifying the correctness of the kinematics forward solution model.

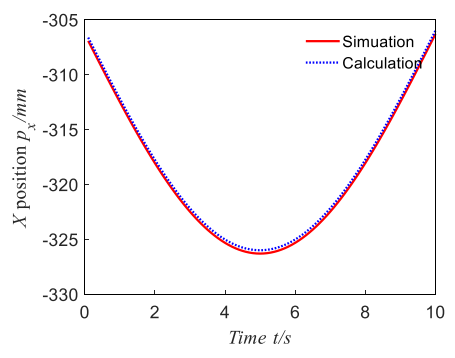

(a)

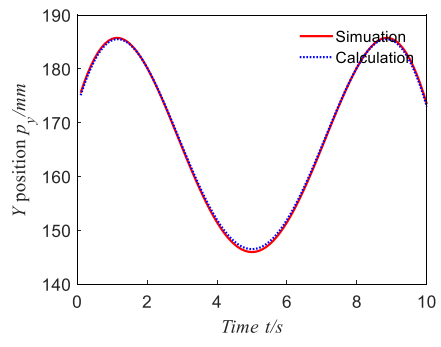

(b) 


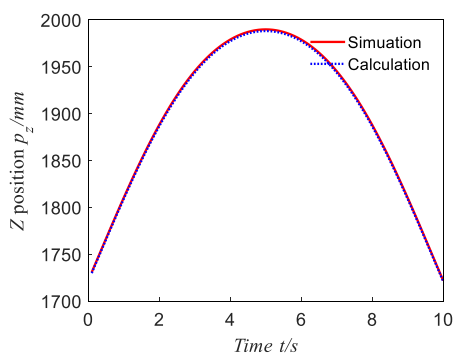

(c)

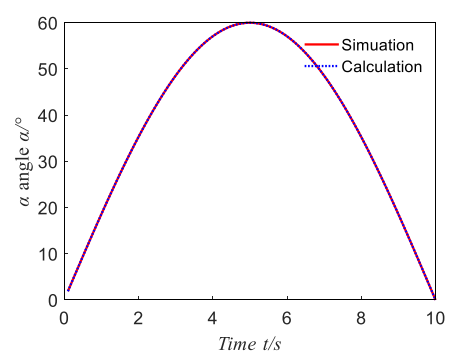

(d)

Fig. 8 Position and orientation curves for the end mechanism. a Curves for $X$-direction; b Curves for $Y$-direction; c Curves for $Z$-direction; d Curves for $\alpha$-angle

\subsection{Inverse kinematics verification}

According to the above analysis, the displacement, $d_{1}$, of the walking mechanism only affected the position of the robot body's workspace, but not the shape of its workspace. Therefore, when verifying the inverse kinematics solution, $\left\{O_{1}\right\}$ was set at the origin of the base standard system, that is, $d_{1}=0$. A parabolic motion in space with an interval of 10 seconds was planned for the mechanism's end device, and the end trajectory of the mechanism could be expressed by Eq. 22:

$$
\left\{\begin{array}{l}
x_{\mathrm{p}}=-t \\
y_{\mathrm{p}}=\frac{1}{2} \cdot t(t-10) \\
z_{\mathrm{p}}=2 \cdot t \\
\alpha=\frac{\pi}{90} \cdot t
\end{array}\right.
$$

In the ADAMS model, the driving function shown in Eq. 22 was applied at the centroid of the mechanism's end device. The $Z_{1}$ moving pair was changed to a fixed constraint for motion simulation, and the displacement data variation rule for the driving pair of each moving joint was output with respect to time through post-processing. Meanwhile, by substituting Eq. 22 into Eq. 20, the displacement variation rule for each moving joint was obtained with respect to time from 
theoretical calculations. The curves generated using both methods are shown in Fig. 9. The figures show that the theoretical calculation results for the kinematic inverse solution had the same variation trend as the results from the software simulation, and the error was very small, thus verifying the correctness of the operational inverse solution model. The figure also shows that the displacement curves for each joint were continuous and smooth during motion, without a mutation phenomenon; in other words, the velocity changes were relatively uniform and the impact was relatively small, meaning that the mechanism had good stability during motion.

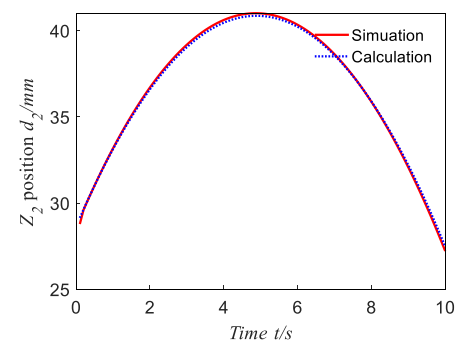

(a)

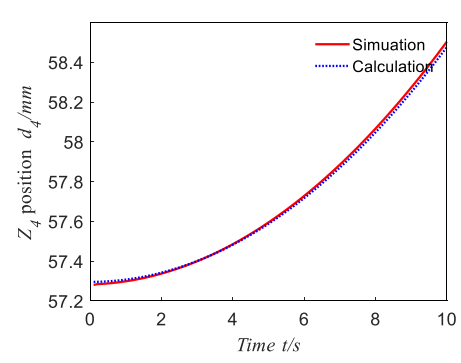

(c)

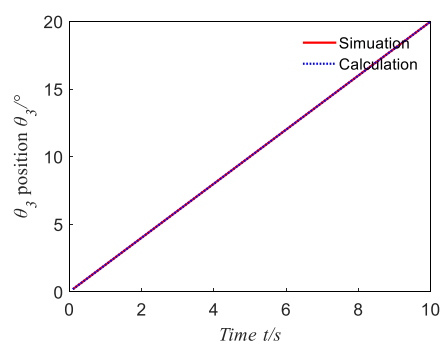

(b)

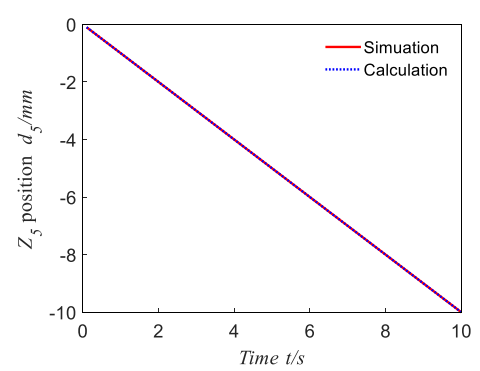

(d)

Fig. 9 Displacement curve for each joint. a Displacement of $\mathrm{Z}_{2}$; b Displacement of $\theta_{3}$; c Displacement of $Z_{4}$; d Displacement of $Z_{5}$

\section{Corrugation trajectory-following experiments}

The robot experimental platform was built according to the mechanism scheme previously described, as shown in Fig. 10. Additionally, ripple-trajectory motion experiments were conducted. 
In the ripple-track motion experiments, the control strategy of uniformly varying the angle climbing the ripple side and the rotation of the ripple top around the virtual rotational center was adopted, and the specific implementation steps are as follows: Firstly, teaching key points in the trajectory and the encoder value of each joint of the teaching point were recorded, and by substituting the encoder values into Eq. 19, the Cartesian coordinates of the terminal device were obtained. Then, trajectory planning was carried out in the Cartesian coordinate space, and by substituting the dense trajectory points obtained after trajectory planning into Eq. 20, the position of each joint in the joint coordinate space was obtained. Finally, the joint motor moves according to the obtained joint space coordinates. The expected normal distance between the terminal device and the ripple surface was set to 0 . The normal error for the whole motion process was dynamically compensated by using LVDT feedback data to fine-tune the $w$-axis guide screw relative to the original position.

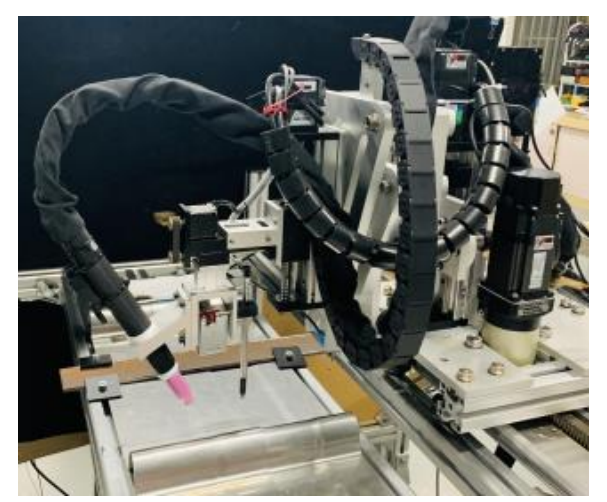

Fig. 10 Robot experimental platform

Ripple-track-following experiments were conducted for two corrugated plates of different specifications, with ripple heights of $47 \mathrm{~mm}$ and $21 \mathrm{~mm}$, respectively. By substituting LVDT data feedback and encoders of each joint into Eq. 19, the terminal device trajectories and axial vectors were drawn and are shown in Fig. 11. The actual trajectory points of the terminal device coincided with the ripple curve, and the vector arrows pointing at the welding gun changed smoothly with the 
ripple curve and maintained a certain angle with respect to the normal direction of the curve.

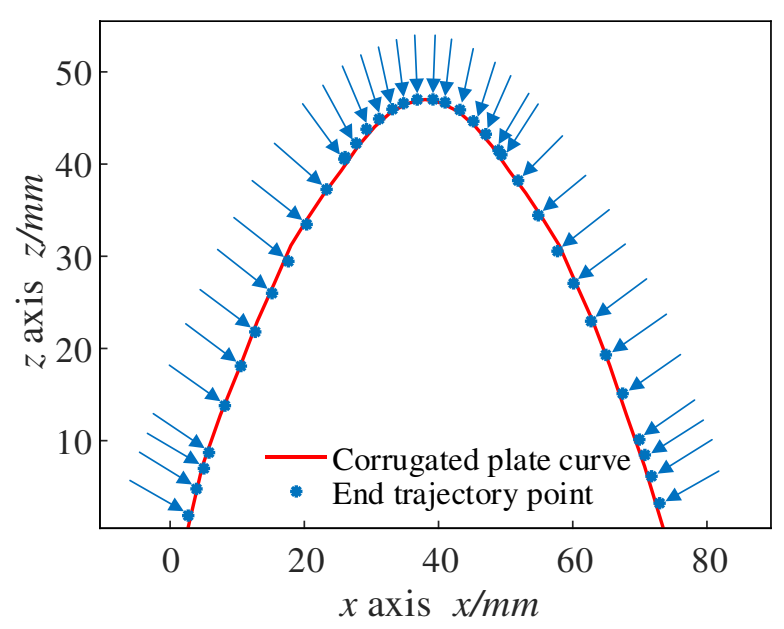

(a)

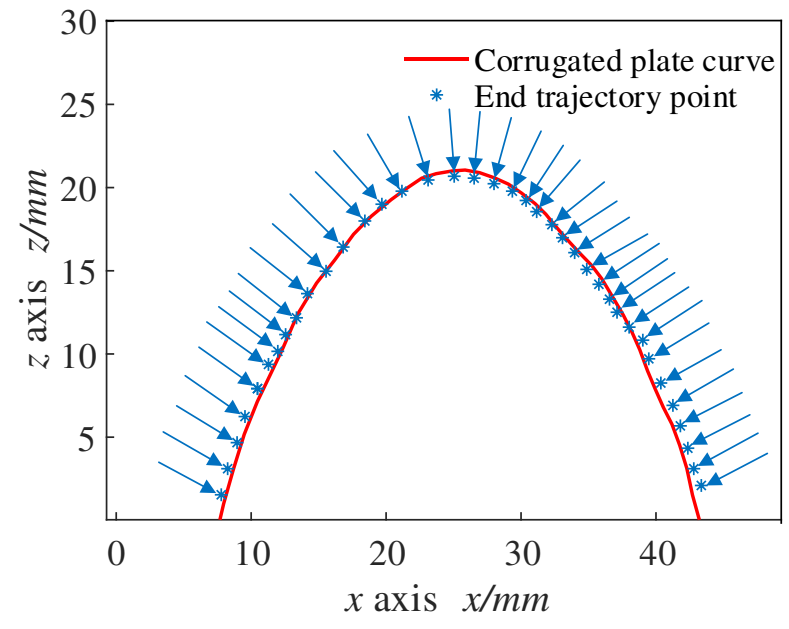

(b)

Fig. 11 End trajectory and axial vector diagrams. a Ripple heights of $47 \mathrm{~mm}$; b Ripple heights of $21 \mathrm{~mm}$

The normal distance deviations between the track points and the corrugated curve were also drawn, as were the angle deviations between the end point and the normal line at that point, as shown in Fig. 12.

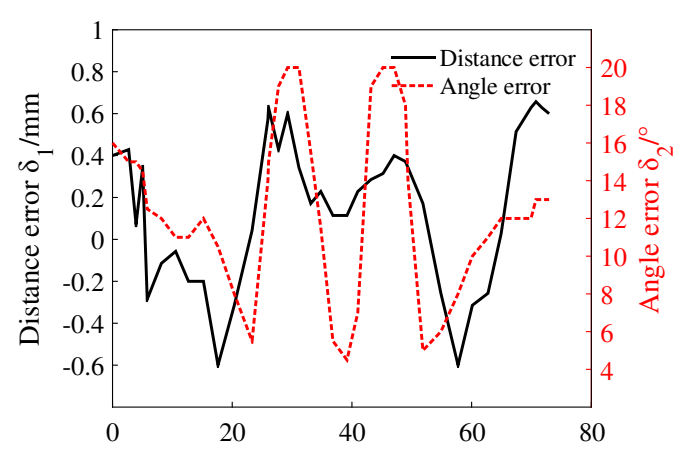

(a)

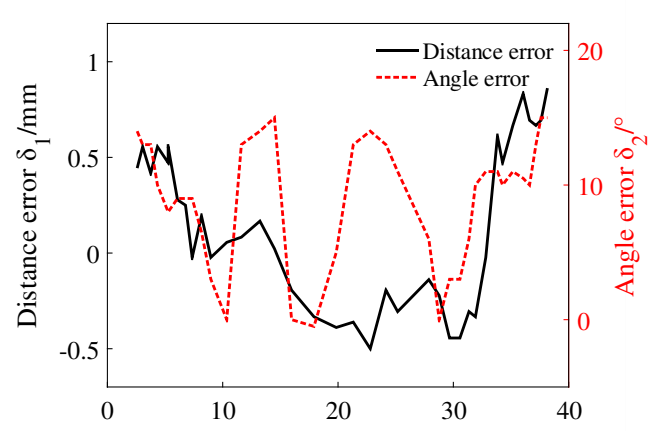

(b)

Fig. 12 Distance and angle deviation curves. a Ripple heights of $47 \mathrm{~mm}$; b Ripple heights of 21 $\mathrm{mm}$

Fig. 12 shows that the maximum distance deviation for the track points was approximately 
$0.8 \mathrm{~mm}$. The angle deviations had approximately symmetrical distributions along the midline of the ripple, and the angle deviation interval was $\in\left(5^{\circ}, 20^{\circ}\right)$. The experimental results show that the welding angle could be controlled within the recommended literature range [19] by setting the initial position of the welding gun to simultaneously meet the welding distance and welding angle requirements for stainless-steel TIG welding.

\section{Conclusions}

Focusing on the problems of mechanism kinematics, workspace design, and welding-process matching during automatic welding of corrugated plates in membrane chambers, a series-parallel-series hybrid automatic welding robot with redundant degrees of freedom and rapid assembly and disassembly was designed.

(1) The composition and configuration characteristics of the robot were analyzed, and the robot degrees of freedom were determined based on screw theory. The kinematics law for the parallel mechanism was analyzed, and forward and inverse solution models for the robot were established. The workspace characteristics of the robot were also analyzed. The results showed that the robot had sufficient adaptability for welding of corrugated plates in membrane tanks.

(2) The simulation and experimental results showed that the robot mechanism was reasonably designed, the motion was smooth, the distance and angle of the end were well controlled, and that the robot mechanism met the requirements for automatic welding of corrugated plates in membrane tanks.

\section{References}

1. Gu Y, Chen X, Wang L (2015) Key technologies for building cargo containment system (CCS) mock-up of membrane type LNG carriers. Nav Archit Ocean Eng 31(2):62-67, 73 
2. Yu YH, Kim BG, Lee DG (2012) Cryogenic reliability of composite insulation panels for liquefied natural gas (LNG) ships. Compos Struct 94(2):462-468. https://doi.org/10.1016/j.compstruct.2011.08.009

3. Ang MH, Lin W, Lim SY (1999) A walk-through programmed robot for welding in shipyards. Ind Rob 26(5):377-388. https://doi.org/10.1108/01439919910284000

4. Zhou B, Liu Y, Xiao Y, Zhou R, Gan Y, Fang F (2021) Intelligent guidance programming of welding robot for 3D curved welding seam. IEEE Access 9:42345-42357. https://doi.org/10.1109/access.2021.3065956

5. Li D, Chen H, Sheng Y, Yang L (2019) Dual-station intelligent welding robot system based on CCD. Meas Sci Technol 30(4):045401. https://doi.org/10.1088/1361-6501/ab02d7

6. Zhang G, Zhang Y, Tuo S, Hou Z, Yang W, Xu Z, Wu Y, Yuan H, Shin K (2021) A novel seam tracking technique with a four-step method and experimental investigation of robotic welding oriented to complex welding seam. Sensors (Basel) 21(9):3067. https://doi.org/10.3390/s21093067

7. Lima EJ, Fortunato Torres GC, Felizardo I, Ramalho Filho FA, Bracarense AQ (2005) Development of a robot for orbital welding. Ind Rob 32(4):321-325. https://doi.org/10.1108/01439910510600182

8. Gui Z, Deng Y, Sheng Z, Xiao T, Li Y, Zhang F, Dong N, Wu J (2014) Design and experimental verification of an intelligent wall-climbing welding robot system. In: 17th International Conference on Climbing and Walking Robots and the Support Technologies for Mobile Machines, Poznan, Poland, 21-23 July 2014. World Scientific, pp 117-124. https://doi.org/10.1142/9789814623353_0014

9. Lee D, Lee S, Ku N, Lim C, Lee K-Y, Kim T-W, Kim J, Kim SH (2010) Development of a 
mobile robotic system for working in the double-hulled structure of a ship. Robot Comput Integr Manuf 26(1):13-23. https://doi.org/10.1016/j.rcim.2009.01.003

10. Ku N, Cha J-h, Lee K-Y, Kim J, Kim T-w, Ha S, Lee D (2010) Development of a mobile welding robot for double-hull structures in shipbuilding. J Mar Sci Technol 15(4):374-385. https://doi.org/10.1007/s00773-010-0099-5

11. Jiang Y (2021) Corrugated plate automatic welding machine and its control system. China Patent 202110952943.4, 12 October 2021

12. Wang Y, Lyu C, Liu J (2021) Kinematic analysis and verification of a new 5-DOF parallel mechanism. Appl Sci 11(17):8157. https://doi.org/10.3390/app11178157

13. Shi X, Ren L, Liao Z, Zhu J, Wang H (2017) Design \& analysis of the mechanical system for a spacial 4-DOF series-parallel hybrid lower limb rehabilitation robot. J Mech Eng 53(13):48-54. https://doi.org/10.3901/jme.2017.13.048

14. Carretero JA, Podhorodeski RP, Nahon MA, Gosselin CM (2000) Kinematic analysis and optimization of a new three degree-of-freedom spatial parallel manipulator. J Mech Des 122(1):17-24. https://doi.org/10.1115/1.533542

15. Guo J, Zhu Z, Chen M, Li Q (2018) Structural design and kinematics modeling of welding robot system for box-type steel structure. Trans China Weld Inst 39(8):32-37, 130. https://doi.org/10.12073/j.hjxb.2018390196

16. Li L, Huang Y, Guo X (2019) Kinematics modelling and experimental analysis of a six-joint manipulator. J Eur Syst Autom 52(5):527-533. https://doi.org/10.18280/jesa.520513

17. Au C, Barnett J, Lim SH, Duke M (2020) Workspace analysis of Cartesian robot system for kiwifruit harvesting. Ind Rob 47(4):503-510. https://doi.org/10.1108/ir-12-2019-0255 
18. Chu W, Huang X, Li S (2021) A calibration method of redundant actuated parallel mechanism for posture adjustment. Ind Rob 48(4):494-509. https://doi.org/10.1108/ir-11-2020-0251

19. Chen Q (2008) Welding handbook. China Machine Press, Beijing

\section{Statements and Declarations}

\section{Funding}

This work was supported by Natural Science Foundation of China [51675233].

\section{Competing Interests}

The authors have no relevant financial or non-financial interests to disclose

\section{Author Contributions}

All authors contributed to the study conception and design. Material preparation, data collection and analysis were performed by all authors. The first draft of the manuscript was written by [Han Qingqing] and all authors commented on previous versions of the manuscript. All authors read and approved the final manuscript. 


\section{Supplementary Files}

This is a list of supplementary files associated with this preprint. Click to download.

- SupplementaryMaterial.rar 